\title{
Distribution of total radiation widths for neutron resonances of $\mathrm{Pt}$ isotopes
}

\author{
P.E. Koehler ${ }^{1,2, a, b}$, F. Bečvář ${ }^{3}$ and M. Krtička ${ }^{3}$ \\ ${ }^{1}$ Los Alamos National Laboratory, Los Alamos, NM 87544, USA \\ ${ }^{2}$ Department of Physics, University of Oslo, N-0316 Oslo, Norway \\ ${ }^{3}$ Charles University in Prague, 18000 Prague 8, Czech Republic
}

\begin{abstract}
High quality neutron capture and transmission data were measured on isotopically enriched ${ }_{192,194,195,196} \mathrm{Pt}$ and natural $\mathrm{Pt}$ samples at ORELA. $R$-matrix analysis of this data revealed resonance parameters for 159, 413, 423, 258, and 11 neutron resonances for neutron energies below 5.0, 16.0, 7.5, 16.0, and 5.0 $\mathrm{keV}$ for ${ }^{192,194,195,196,198} \mathrm{Pt}+\mathrm{n}$, respectively. Earlier analysis of data on reduced neutron widths, $\Gamma_{n}^{0}$, showed that the distributions of $\Gamma_{n}^{0}$ for ${ }^{192,194} \mathrm{Pt}$ deviate significantly from the Porter-Thomas distribution (PTD) predicted by random matrix theory. In this contribution we report on preliminary results of the analysis of distribution of total radiation widths, $\Gamma_{\gamma}$, in ${ }^{192,194,195,196} \mathrm{Pt}+\mathrm{n}$ reactions. Comparison of experimental data with predictions made within the nuclear statistical model indicates that standard models of Photon Strength Functions (PSFs) and Nuclear Level Density predict $\Gamma_{\gamma}$ distributions which are too narrow. We found that satisfactory agreement between experimental and simulated distributions can be obtained only by a strong suppression of the PSFs at low $\gamma$-ray energies and/or by violation of the usual assumption that primary transitions from neutron resonances follow the PTD. The shape of PSFs needed for reproduction of our $\Gamma_{\gamma}$ data also nicely reproduces spectra from several $(\mathrm{n}, \gamma)$ experiments on the neighbor nuclide ${ }^{198} \mathrm{Au}$.
\end{abstract}

\section{Introduction}

An analysis of high-resolution time-of-flight data on neutron cross sections of ${ }^{95} \mathrm{Mo}$ [1] demonstrated that distribution of total radiation widths $\Gamma_{\gamma}$ of neutron resonances carries valuable information for testing the validity of the statistical model and its input photon strength functions (PSFs). This finding motivated us to undertake a statistical analysis of $\Gamma_{\gamma}$ distributions of unprecedentedly large sets of resonances that we observed for ${ }^{192,194,195,196} \mathrm{Pt}+\mathrm{n}$ systems [2,3]. An added motivation was that such simulations might shed more light on the origin of deviations of ${ }^{192,194} \mathrm{Pt} \Gamma_{n}^{0}$ values for these same resonances from the expected Porter-Thomas distribution [2]. Also, the shapes of PSFs in the mass region near $A=200$ have been the subject of debate for more than 50 years. For example, anomalous behavior of PSFs in this mass region was observed from thermal neutron capture many years ago [6]. Attempts to verify the most prominent anomaly in ${ }^{198} \mathrm{Au}$ met with variable success. However, recent $(\mathrm{n}, \gamma)[7]$ and ${ }^{3} \mathrm{He}$-induced photon production experiments [8] indicate the presence of a marked wiggle in the PSF of ${ }^{198} \mathrm{Au}$. It is presently not clear whether this structure is an artifact of a pygmy resonance or a mere irregularity of the PSF having no link to any nuclear vibrational mode. Fluctuation analysis of $\Gamma_{\gamma}$ of Pt isotopes may, in principle, contribute to clarify-

\footnotetext{
a e-mail: paul.koehler.1@us.af.mil

${ }^{\mathrm{b}}$ Current address: Air Force Technical Applications Center, Patrick Air Force Base, Florida, USA
}

ing this problem. Only preliminary results are presented herein.

\section{Experiment and Data reduction}

The time-of-flight method was used to make both neutron transmission and capture measurements at the Oak Ridge Electron Linear Accelerator (ORELA) on a set of enriched Pt samples. Experiment details can be found in Refs. [4, 5].

Neutron capture measurements were made at a sourceto-sample distance of $40.12 \mathrm{~m}$ using a pair of $\mathrm{C}_{6} \mathrm{D}_{6}$ detectors, and employed the pulse-height-weighting technique. Total neutron cross sections were measured via transmission using a ${ }^{6} \mathrm{Li}$-loaded glass scintillator at a source-todetector distance of $79.827 \mathrm{~m}$.

The $R$-matrix code SAMMY was used for simultaneous fitting of all the transmission and capture data to extract resonance parameters, including $\Gamma_{\gamma}$, at neutron energies below 5.0, 16.0, 7.5, 16.0, and $5.0 \mathrm{keV}$ for $192,194,195,196,198 \mathrm{Pt}$, respectively. Overall, 1264 resonances for these nuclides were analyzed.

The shape of strong resonances in the neutron transmission data allowed determination of neutron orbital momentum. Present analysis of $\Gamma_{\gamma}$ data was confined to resonances having both firm $s$-wave assignments and statistical uncertainties less than $10 \%$. The $\Gamma_{\gamma}$ distributions for each isotope were found to be similar, displaying a marked asymmetry. 

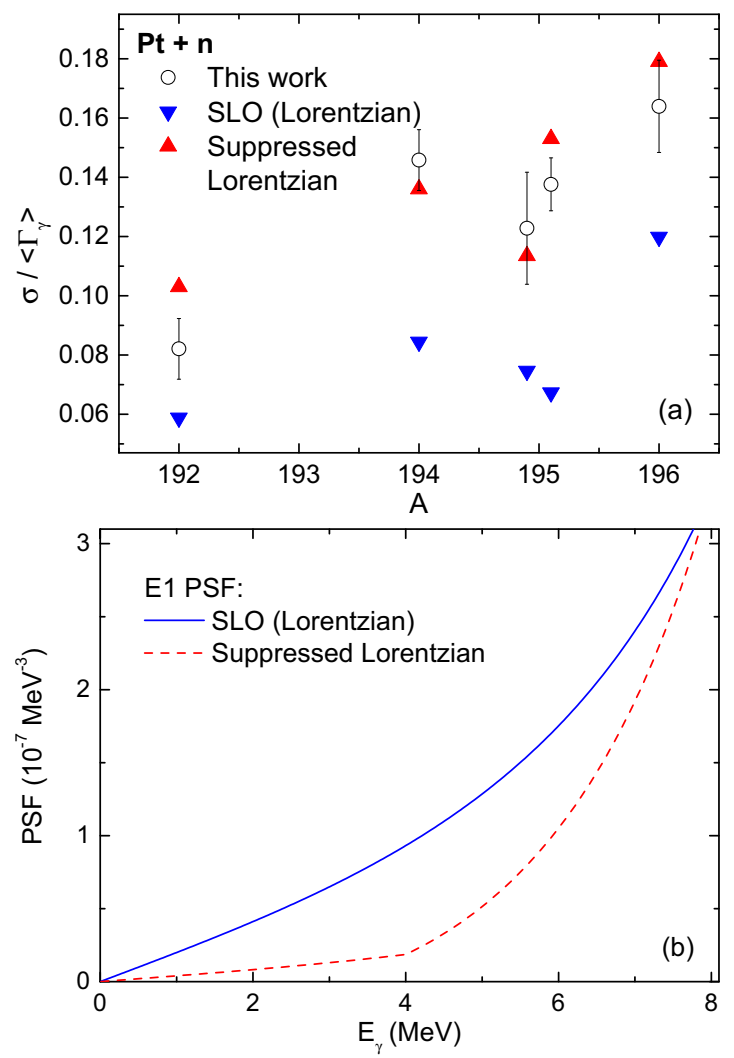

Figure 1. Relative widths of the distributions of $\Gamma_{\gamma}$ for Pt isotopes obtained from a Gaussian fit (a). The energy dependences of $E 1$ PSF models used in simulations (b). The SLO model for $E 1$ PSF in combination with the CT model for level density, used in presented simulations, gives the widest distribution of $\Gamma_{\gamma}$ out of all conventional models, but still falls short of agreeing with our data. Simulations using a suppressed Lorentzian E1 PSF result in much better agreement with our data.

\section{Data Analysis}

According to the statistical model, partial radiation widths for transitions between an initial level $i$ and a final level $f$ of type $X$ (electric or magnetic) and multipolarity $L$ follow the Porter-Thomas distribution (PTD) with expectation values $\left\langle\Gamma_{i \gamma f}\right\rangle=f_{X L}\left(E_{\gamma}\right) E_{\gamma}^{2 L+1} / \rho\left(E_{i}, J_{i}, \pi_{i}\right)$, where $E_{\gamma}$ is the $\gamma$-ray energy, $\rho$ is the density of initial resonances with a given spin and parity at energies $E_{i}$, while $f_{X L}\left(E_{\gamma}\right)$ is the PSF for corresponding transition type. The total radiation width $\Gamma_{\gamma}$ of a neutron resonance is given by the sum of partial radiation widths for transitions to all levels $f$ below the neutron capturing state $\Gamma_{\gamma}=\sum_{f} \sum_{X L} \Gamma_{i \gamma f}(X L)$.

A total radiation width can be considered to be a random variable resulting from convolution of a large number of independent variables with different expectation values governed by the PTD. In view of this, even in conditions when the level density and PSFs are precisely known, there is no straightforward way for predicting the shape of the distribution of this variable. To overcome this problem, Monte Carlo approach can be used to simulate the distribution. In heavy nuclei, the resultant distribution is expected to be very close to a $\chi^{2}$ distribution with a large number of degrees of freedom. Furthermore, such a $\chi^{2}$ distribution can in turn be closely approximated by a Gaussian distribution. The ratios of Gaussian means $\left(\left\langle\Gamma_{\gamma}\right\rangle\right)$ and standard deviations $(\sigma)$ from maximum-likelihood analyses of our data are shown in Fig. 1.

Our Monte Carlo simulations using conventional models for level density (BSFG or CT models, see [9]) and PSFs (SLO, KMF or GLO models of E1 PSF and spin-flip or single-particle models of $M 1$, see [9]) resulted in distributions much narrower than our data, as shown in Fig. 1. In contrast, satisfactory agreement between our measured and simulated $\Gamma_{\gamma}$ distributions can be obtained by a strong suppression of the PSFs at low $\gamma$-ray energies, similar to that found to be needed to explain data for the neighbor ${ }^{198} \mathrm{Au}$ nuclide [7]. Broader $\Gamma_{\gamma}$ distributions also can be obtained by assuming that primary transitions from neutron resonances do not follow the PTD but instead have a broader distribution as was observed for ${ }^{192,194} \mathrm{Pt} \Gamma_{n}^{0}$ values of the same resonances.

In general, these results cast doubt on the validity of the statistical model or at least conventional PSF models, and could have impact on research in the field of $\gamma$ decay of highly-excited nuclear levels and in nuclear astrophysics. More advanced comparisons of observed and simulated $\Gamma_{\gamma}$ distributions are planned.

\section{Acknowledgements}

This work was supported by the Research Council of Norway, by the Nuclear Criticality Safety Program, by the Office of Nuclear Physics of the US Department of Energy, and by the grant No. 13-07117S of the Czech Science Foundation.

\section{References}

[1] P.E. Koehler et al., Phys.Rev. C 88, 041305(R) (2013)

[2] P.E. Koehler et al., Phys.Rev. Lett. 105, 072502 (2010)

[3] P.E. Koehler et al., to be published

[4] P.E. Koehler et al., Nucl. Phys. A 688, 86c (2001)

[5] P.E. Koehler and K.H. Guber, Phys. Rev C 88, 035802 (2013)

[6] G.A. Bartholomew, B.B. Kinsey, Canad. J. Phys. 79, 1025 (1953)

[7] M. Krtička et al., AIP Conference Proceedings 831, 481 (2006)

[8] F. Giacoppo et al., EPJ Web Conferences 66, 02041 (2014)

[9] R. Capote et al., Nucl. Data Sheets 110, 3107 (2009) 